\title{
Patterned delivery and expression of gene constructs into zebrafish embryos using microfabricated interfaces
}

\author{
Tushar Bansal • Justin Lenhart • Taesung Kim • \\ Cunming Duan • Michel M. Maharbiz
}

Published online: 9 January 2009

(C) The Author(s) 2008. This article is published with open access at Springerlink.com

\begin{abstract}
We demonstrate a method which uses simple microfabrication and microfluidics to produce custom, shaped electroporators for the patterned delivery of foreign molecules into developing embryos. We show how these electroporators can be used to 'draw' two-dimensional patterns of tracer molecules, DNA and mRNA into the yolk and cells of zebrafish embryos (Danio rerio) at different stages of development. We demonstrate the successful delivery of patterns of Trypan Blue (normal dye), Texas Red (fluorescent dye), GFP-expressing DNA plasmids and GFP expressing mRNA constructs into both chorionated and dechorionated embryos. Both DNA and mRNA were expressed in the desired patterns subsequent to delivery. Square pulses of $10-20 \mathrm{~V}(0.20-0.40 \mathrm{kV} / \mathrm{cm}), 50-100 \mathrm{~ms}$ width were sufficient to create transient pores and introduce compounds from the late blastula period ( $3 \mathrm{hpf}$ ) to early pharyngula period ( $24 \mathrm{hpf}$ ) embryos. Using $24 \mathrm{hpf}$ dechorionated embryos, we achieved a high survival of $91.3 \%$ and
\end{abstract}

Electronic Supplementary Material The online version of this article (doi:10.1007/s10544-008-9273-5) contains supplementary material, which is available to authorized users.

T. Bansal $(\triangle)$

Department of Electrical Engineering and Computer Science,

University of Michigan,

Ann Arbor, MI, USA

e-mail: tbansal@umich.edu

J. Lenhart $\cdot$ C. Duan

Department of Molecular, Cellular and Developmental Biology, University of Michigan,

Ann Arbor, MI, USA

T. Kim $\cdot$ M. M. Maharbiz

Department of Electrical Engineering and Computer Science, University of California,

Berkeley, CA, USA
$89 \%$, and a delivery efficiency of $38 \%$ and $50 \%$ for GFPDNA and GFP-mRNA respectively. Lastly, we demonstrate the simultaneous delivery of different compounds into the developing embryo.

Keywords Spatio-temporal · Electroporation - DNA . Microfabricated · Zebrafish

\section{Introduction}

There is a considerable interest in the patterned chemical intervention of developing organisms (Lucchetta et al. 2005; Ismagilov and Maharbiz 2007). The ability to modify spatiotemporal gene expression in two (or three) dimensions via patterned delivery of genes, proteins and bioactive molecules would allow for more biologically-relevant manipulations of developing systems. For example, Voiculescu et al. (2007, 2008) recently demonstrated spatialtemporal control of DNA and morpholino oligonucleotide (MO) doses in selected regions of early chick embryos with a custom electroporation setup made from hand-modified platinum wires and inserted into an acrylic fixture. Several new chemical methods, such as the tetracycline, dexamethasone and insect hormone regulated gene expression systems would benefit from a method which provides patterned delivery of trigger compounds into developing embryos (Shestopalov and Chen 2008). Similarly, simultaneous delivery of multiple molecules in different patterns would also expand the type of experiments performed in spatially heterogeneous systems, particularly where multiple pathways with specific timings are involved (Ismagilov and Maharbiz 2007; Kawakami et al. 2005; Vonk and Richardson 2008); see for example, Mara et al. (2007) which demonstrates zebrafish somitogenesis is governed by 
a segmentation clock that generates oscillations in expression of several Notch pathway genes. In this system, chevron-shaped areas of gene expression within the tailbud demarcate where the clock is set up in the progenitor cells (priming), where the clock starts running (initiation), and where the clocks of neighboring cells are entrained (synchronization).

A number of microfabricated electroporators for cell culture use have recently emerged (Kim et al. 2007; Huang and Rubinsky 1999). With respect to embryos, most current electroporation techniques result in whole organism dosing as transient pores are created throughout the organism (Bosch et al. 2002) or, in the case of older embryos, in single point source delivery similar to microinjection. For either technique mentioned above, the addition of molecules into patterns other than single points, is limited. Various groups are starting to build microsystem platforms to introduce compounds into single cells and developing embryos (Khine et al. 2005; Zappe et al. 2006). Huang et al. (2007) introduced the first microfabricated device to electroporate zebrafish embryos. Huang's technology reproduced the capabilities of conventional electroporators in chorionated embryos but with limited resolution. Cerda et al. (2006) recently published a protocol to electroporate DNA, RNA and morpholinos in zebrafish embryos; their study first performed a single-point microinjection followed by electroporation to improve delivery efficiency. Other novel techniques utilizing laser systems with conventional equipment (Kohli et al. 2007) have also been recently demonstrated but remain difficult to replicate.

This paper demonstrates the combination of electroporation and microfluidics (without microinjection) to deliver foreign compounds into zebrafish embryos with specific patterns in localized regions. We demonstrate the efficacy of our chip by using dechorionated and chorionated as well as young ( $3 \mathrm{hpf}$ ) and old ( $24 \mathrm{hpf}$ ) embryos as measured in hours post fertilization (hpf). Additionally, we achieved successful delivery in both yolk and cells of the zebrafish. Platinum electrodes were microfabricated into various shapes with lithography and passivated with silicone elastomer. In most cases, single square pulses of $10-20 \mathrm{~V}(0.20-0.40 \mathrm{kV} / \mathrm{cm}), 50-100 \mathrm{~ms}$ pulse width were provided by a commercial function generator and were sufficient to create transient pores and introduce compounds in late blastula period to early pharyngula period embryos. Trypan blue and Texas red were initially electroporated into the yolk. Using $24 \mathrm{hpf}$ dechorionated embryos, we achieved a high survival of $91.3 \%$ and $89 \%$ and a delivery efficiency of $38 \%$ and $50 \%$ for GFP-DNA and GFP-mRNA respectively. We believe that our simple technique offers the unique advantage of introducing foreign compounds at local sites and in specific patterns unlike any other microsystem technique and provides a new tool to aid advanced studies in cellular development and morphogenesis.

\section{Materials and methods}

\subsection{Device theory}

During electroporation, transient pores are created in a cell membrane when short electrical pulses (exceeding the membrane's dielectric strength) are applied. While a pore is open, extracellular compounds in the vicinity of the pore can enter and transit into the cell interior. During conventional electroporation, a high concentration of cells and relevant molecules (dyes, proteins, genes, etc.) in suspension are introduced between two opposite electrodes. A high electric field, typically from 1 to $10 \mathrm{kV} / \mathrm{cm}$, is applied to the electrodes to deliver the molecules into the cells (Huang et al. 2007; Rambabu et al. 2005; Lurquin 1997). Electroporation is a well-characterized method for molecule delivery into embryos of many species (Lurquin 1997; Swartz et al. 2001), although microinjection is more common for zebrafish embryos. As might be expected, compound trans-membrane permeability is highest for cells nearest to the electrodes (Rambabu et al. 2005). The amplitude and frequency of the applied potential, as well as its overall shape and total duration are usually empirically determined for each cell type; a range exists wherein membrane pores reseal themselves after delivery (Rambabu et al. 2005). Excessive field strength or long durations cause irreversible damage to the cells and the organism (Lurquin 1997).

\subsection{Device preparation}

All devices were fabricated on 4" glass substrates (Supplementary Fig. 1). Wafers were cleaned in Piranha $\left(1: 1 \mathrm{H}_{2} \mathrm{SO}_{4} / \mathrm{H}_{2} \mathrm{O}_{2}\right)$ for 20 min followed by a 10 -min rinsedry cycle. Photoresist was then spun on, patterned into electrode geometries and soft-baked. A descum was performed for $3 \mathrm{~min}$ at $80 \mathrm{~W}$ to remove any residual traces of photoresist; this enhances the lift-off yield significantly. Titanium $(500 \AA)$ and platinum $(1,000 \AA)$ were deposited using evaporation and patterned using a lift-off process. Parts of the metal areas were then passivated with Polydimethylsiloxane (PDMS) acting as the silicone elastomer, leaving only the relevant metal shapes exposed to electric fields. This passivation can be performed by either (a) spinning and curing Sylgard ${ }^{\circledR} 184$ and cutting out windows in the cured polymer using a sharp blade or (b) spinning $(300 \mathrm{rpm})$, baking $\left(130^{\circ} \mathrm{C}, 2 \mathrm{~min}\right)$, lithographically exposing and curing $\left(150^{\circ} \mathrm{C}, 2 \mathrm{~min}\right)$ Dow Corning ${ }^{\circledR}$ WL-5150 photo-patternable silicone (according to manufacturer's instructions) to pattern appropriate windows in 


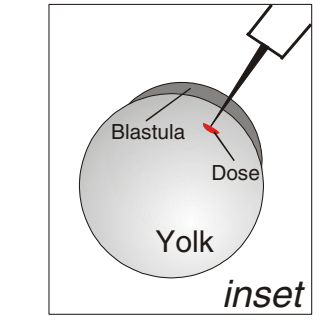

(A)

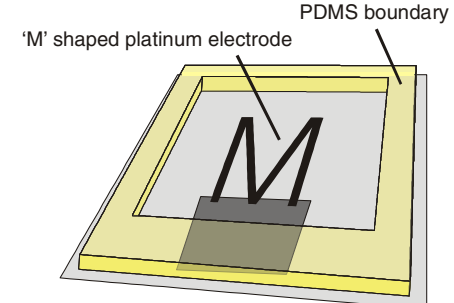

(B)

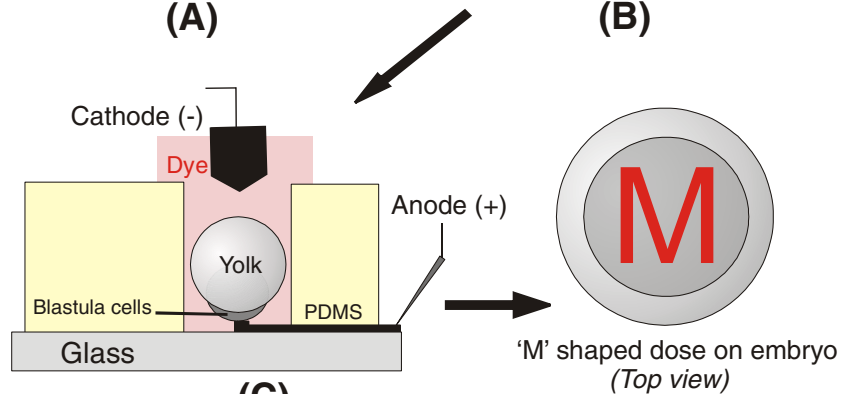

(C) (Top view)
Fig. 1 Conceptual zebrafish electroporation setup is shown. (a) Microinjection remains a common but labor intensive process. (b) Microscale noble metal electrodes were fabricated on a glass substrate in our device. These electrodes acted as anodes during electroporation. (c) During dosing, embryos were placed on top of the patterned anode. The PDMS boundary created a chamber which contained the dye solution. A second electrode on top of the embryo acted as cathode. On applying short electric pulses between electrodes (electroporation), electrode-shaped patterns of extracellular compounds were delivered into the embryo

the polymer. The resultant setup effectively shields electric fields and forms a chamber around the electrode to contain the solution (PDMS is hydrophobic) and the embryo.

A second set of multi-compound dosing devices were also constructed. Metal microfluidic channels were fabricated over the electrodes via a sacrificial release method and micropores were wet etched in the channel roof. These devices could deliver multiple chemicals simultaneously into the embryo. A detailed fabrication process is provided in Supplementary Fig. 2.

\subsection{Zebrafish rearing and preparation}

Zebrafish embryos obtained from the Duan lab were grown according to the procedure in (Westerfield 2000), maintained on a 14-10 h light dark cycle. A stock solution of E3 medium was prepared $(5 \mathrm{mM} \mathrm{NaCl}, 0.17 \mathrm{mM} \mathrm{KCl}$, $0.33 \mathrm{mM} \mathrm{CaCl}_{2}, 0.33 \mathrm{mM} \mathrm{MgSO}$, and $0.1 \%$ methylene blue) and once embryos were obtained subsequent to mating (usually daily), they were transferred in this medium and incubated at $28.5^{\circ} \mathrm{C}$. Embryos were de-chorionated by mixing in $125 \mu \mathrm{g} / \mathrm{ml}$ of Pronase (Roche) in E3 medium and gently shaking the Petri-dish until the chorion came apart. They were rinsed thrice to remove all the pronase. Just before electroporation, embryos were anaesthetized by using MS222 (Sigma). During electroporation, embryos were held immobile within the PDMS wall chamber. They were gently pippetted into that chamber and positioned in the proper orientation over the stimulating electrodes using $5 \mathrm{~A}$ tweezers and gentle pushing or using rolling motions. Methyl cellulose (Sigma) was also tested to hold embryos immobile, but slowed throughput and did not provide a significant advantage toward proper positioning of the embryo with respect to the electrodes.

\subsection{Dye preparation}

For fluorescent imaging and dosing, solutions were prepared by mixing E3 medium with appropriate dyes. These dyes were chosen due to their low molecular weight. $30 \mathrm{mM}$ solutions of Texas $\operatorname{Red}^{\circledR}$ Dextran 3,000 MW, lysine fixable (Invitrogen) and Fluorescein were prepared. A $0.4 \%$ (by volume) trypan blue (Gibco) solution was also prepared.

\subsection{DNA and mRNA preparation}

pCS2eGFP vectors were prepared according to the procedure outlined by Dave Turner and Ralph Rupp (Rupp et al. 1994; Turner and Weintraub 1994). Briefly, A DNA fragment containing the Kozak sequence followed by entire ORF of EGFP (Clontech, USA) was generated by PCR. This DNA fragment was subcloned into the pCS2+ vector to generate the EGFP overexpression plasmid DNA (pCS2+EGFP). The purified plasmid was dissolved in DNase free water and stored at $-20^{\circ} \mathrm{C}$ until use. The pCS2+EGFP plasmid was linearized by restriction enzyme (Notl) digestion and was used for capped EGFP mRNA synthesis. Capped RNA was synthesized by in vitro mRNA transcription using mMassage mMachine kit (Ambion, TX). Prepared EGFP mRNA was dissolved in diethylpyrocarbonate (Sigma, USA) treated water and kept at $-80^{\circ} \mathrm{C}$ until use.

\subsection{Instrumentation}

GFP-DNA and GFP-mRNA imaging was done by Leica MZ 16F upright microscope. Adobe Photoshop and ImageJ were used to process figures. Upright and inverted microscopy was done with ZEISS Axioshop and a Nikon TE2000, respectively for dye imaging. Electroporation potential was applied with an Agilent 33220A Function/ Arbitrary Waveform Generator, $20 \mathrm{MHz}$. Single square wave pulses of $10-20 \mathrm{~V}, 50-100 \mathrm{~ms}$ pulse width was applied between the electrodes.

\subsection{Experimental setup}

Figure 1 illustrates the experimental setup. During conventional electroporation in most organisms, electrodes are 

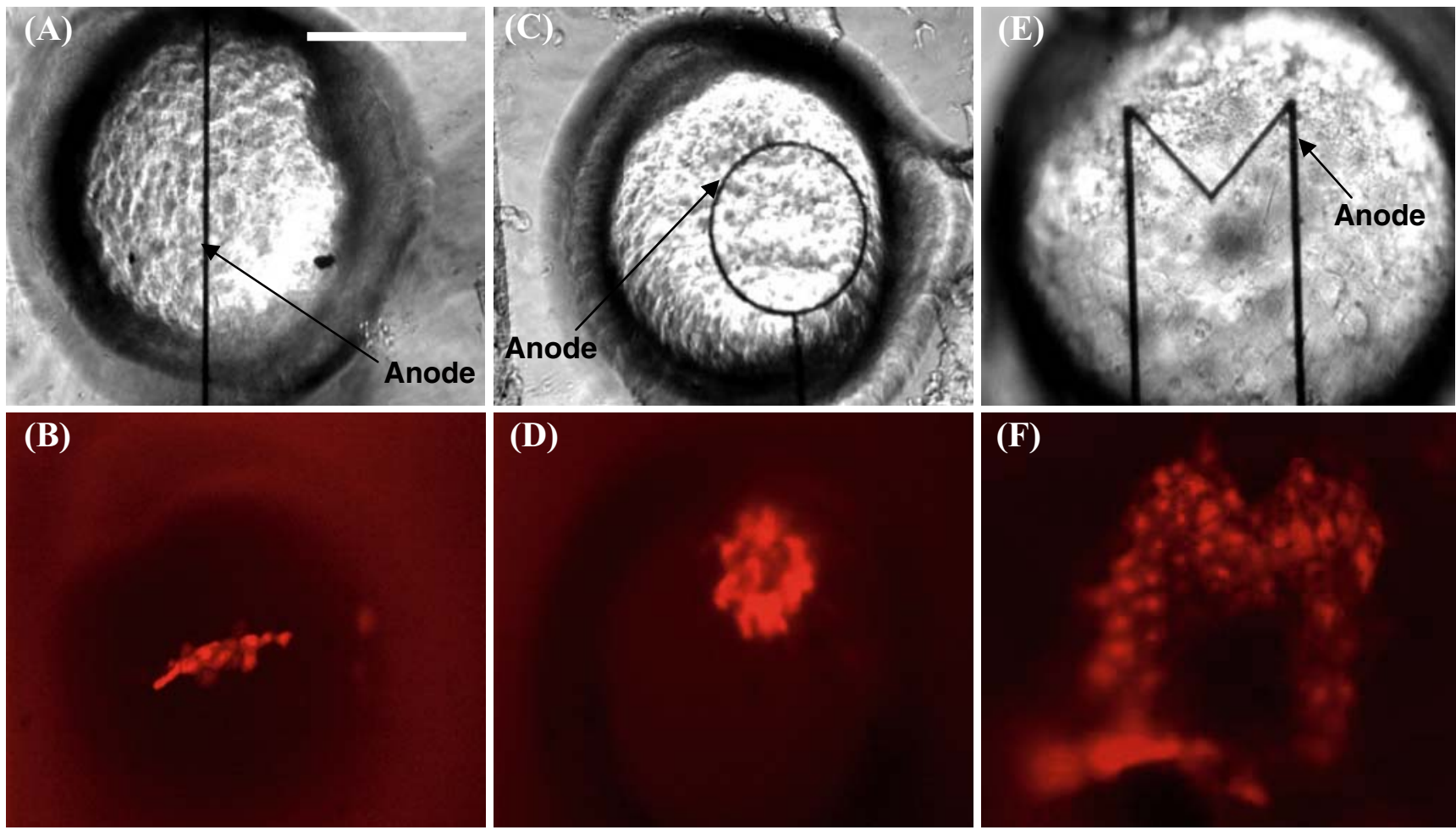

Fig. 2 Fluorescent texas red was electroporated in various shapes in the yolk of zebrafish embryos at $10 \mathrm{hpf} .10 \mu \mathrm{m}$ wide electrodes were designed in various shapes and used as anodes. (a) A white light image of a line electrode with embryo on top. (b) A fluorescent image of a line electrode having been electroporated into the yolk (Once the embryos were pipetted into a clean dish for fluorescent imaging, they were not touched and hence are not oriented to the white light image) (c) A white light image of an 'O' ring pattern. (d) Fluorescent image of an ' $\mathrm{O}$ ' ring. (e) White light image of ' $\mathrm{M}$ ' shaped design. (f) Fluorescent image of the ' $\mathrm{M}$ ' pattern. Two square pulses of $10 \mathrm{~V}$, $50 \mathrm{~ms}$ pulse width were used. Scale bar is $200 \mu \mathrm{m}$ placed on both sides of the embryo and transient pores are created by providing electrical pulses between them. This creates pores in the entire organism. In zebrafish embryos, however, microinjection is first used to deliver extracellular compounds locally (Fig. 1(a)) followed by electroporation of the entire embryo to enhance the microinjection results.

In our setup, lithographically-patterned metal (Fig. 1(b)) on thin glass acted as the anode and a distant metal wire was placed in solution approximately $500 \mu \mathrm{m}$ away and acted as the cathode (these roles were switched during negatively charged pCS2eGFP DNA electroporation delivery). PDMS was used both as an insulator and as a boundary around the anode to create a chamber and contain the movement of the embryo.

During an experiment, the chamber was filled with embryo medium mixed with the appropriate delivery molecule. In the case of multi-compound dosing devices, the microfluidic channels were first filled with the appropriate dyes (and no compound was introduced into the bulk medium). Embryos were then pipetted into the chamber where they settled on top the electrode. The cathode was lowered on top of the embryo at this time. A commercial voltage source (Agilent 33220A) was used to deliver square wave pulses of $10-20 \mathrm{~V}, 50$
$100 \mathrm{~ms}$ pulse width between the electrodes. After the pulse was applied and electroporation conducted, embryos were gently removed from the device with a pipette and moved into a Petri dish containing E3 medium and incubated in $28.5^{\circ} \mathrm{C}$ for $24 \mathrm{~h}$.

\section{Results and discussion}

As with any electroporation protocol, long duration and high voltage pulses can kill cells (Cerda et al. 2006; Sale and Hamilton 1967, 1968). The ideal voltage, pulse length and number of pulses is difficult to determine and changes for each setup. Here, we first determined these values experimentally (data not shown) by attempting a range of known good values (Cerda et al. 2006) with a simple, straight electrode. These parameters (see below) were then used throughout the experiment.

\subsection{Dye delivery}

As a benchmark of basic electroporation, Trypan blue dye was electroporated in early stage (3-5 hpf) embryos with 
intact chorion. Two square pulse of $20 \mathrm{~V}, 100 \mathrm{~ms}$ pulse width, $1 \mathrm{~s}$ apart were used. Electroporation created temporary pores in the chorion which enabled trypan blue to readily diffuse into the perivitelline space of the embryos (Supplementary Fig. 3). To reduce the adhesion effect of trypan blue, the embryos were kept in E3 solution for $3 \mathrm{hrs}$ before being imaged (Cerda et al. 2006). Control embryos $(V=0)$ were used as reference.

Next we used the devices to dose patterns of a fluorescent tracer dye (Texas Red) commonly used when tracking cell fate in embryos. Figure 2(a) shows a $10 \mathrm{hpf}$ zebrafish embryo on top of a single, long $10 \mu \mathrm{m}$ wide fabricated platinum anode. The embryo was aligned such that the yolk was in direct contact with the anode while the cathode was $500 \mu \mathrm{m}$ away. Two square pulses of $10 \mathrm{~V}$, $50 \mathrm{~ms}$ pulse width, $1 \mathrm{~s}$ apart were used. Figure 2(b) shows the fluorescent image of the embryo with the pattern of a line delivered in the yolk. Cells closer to the electrode tended to have sharper features. Only part of the line was imprinted onto the embryo due to the curvature of the yolk (Supplementary Fig. 4). In Figs. 2(c) and (d), an ' $O$ ' shaped electrode with a width of $15 \mu \mathrm{m}$ was electroporated onto the embryo under the same conditions. Other patterns such as the letter ' $M$ ' (Fig. 2(e), (f)), multiple lines (Supplementary Fig. 5(a), (b)) and single dots (Supplementary Fig. 5(c), (d)) were also delivered.

\subsection{DNA and mRNA delivery}

To demonstrate the successful delivery of biologically active molecules with our device, we first electroporated GFP-DNA and GFP-mRNA vectors into young zebrafish embryos and recorded the resultant expression of green fluorescent protein (GFP). In Supplementary Fig. 6(a) and (b), $0.250 \mu \mathrm{g} / \mu \mathrm{l}$ solution of pCS2eGFP DNA was pipetted in the chamber and a $3 \mathrm{hpf}$ embryo was placed on the electrode. Electroporation was performed and pCS2eGFP DNA entered the cells where it integrated with the nucleus and expressed itself. Supplementary Fig. 6(c) shows the fluorescence from GFP at $24 \mathrm{hpf}$ expressed by the DNA sequestered inside dosed cells. No patterns could be formed on blastula stage cells as it takes $4-6 \mathrm{hrs}$ for the DNA to integrate itself with the zebrafish and any pattern was lost during this time as cells divide continuously. Embryos treated in this way developed normally into fish.

We then used the above setup to electroporate patterned lines both at the yolk sac and at the brain region of older developing zebrafish. A single square pulse of $10 \mathrm{~V}$, $100 \mathrm{~ms}$ pulse width was sufficient to deliver DNA into zebrafish at $24 \mathrm{hpf}$. Figure 3 shows the same patterned electrode being used to deliver DNA-GFP in the shape of a line to a $24 \mathrm{hpf}$ embryo. Figure 3(a) and (b) represent white light and fluorescent images of zebrafish at 48 hpf. A gap of $24 \mathrm{~h}$ ensured proper integration of DNA to the nucleus. The dotted red lines indicate the position of the cathode during dosing. In this case, the cathode was aligned parallel to the anterior-posterior axis of the zebrafish. Fig. 3(c) and (d) present a close-up top view of the same fish. Figure 3(e) and (f) present white light and fluorescent images of a second zebrafish sample. In this case, the cathode was aligned perpendicular to anterior-posterior axis. Zebrafish dosed with DNA-GFP always exhibited the mosaic expression seen in Fig. 3(b), (d), and (f) in which some but not all cells along the pattern expressed GFP. This was expected and correlates with whole-embryo electroporation (where not all cells are known to express the dosed genetic material). This is usually attributed to the fact that injected DNA, unlike injected mRNA, must enter not just the cell membrane, but also the cell nucleus and be transcribed efficiently. It is generally assumed that this mosaic pattern is due to the DNA being excluded from the nuclei of the non-expressing cells (Hogan et al. 1994). Increased expression percentage can be achieved by using a higher concentration of DNA but excessive DNA can lead to increased cell toxicity. Alternatively, there are known sequences (virus- or transposon-related factors) that can increase the merging percentage of the chromosomal DNA and dramatically increase the uniformity of the pattern (less mosaic) (Hogan et al. 1994). We also demonstrated how the devices could be used to deliver single points in a developing embryo (as with conventional electroporation or microinjection). In Supplementary Fig. 7(a), the majority of the cathode was passivated with PDMS, exposing only a small $10 \mu \mathrm{m} \times 10 \mu \mathrm{m}$ area at the tip. The expression from the resultant dosed cell is shown in Supplementary Fig. 7(b) and (c).

Introduction of in vitro transcribed mRNA is a common technique to gain insight into the in-vivo activity of newly isolated cDNAs and has been the most commonly used method to express proteins in zebrafish embryos (Hogan et al. 1994; Kane and Kimmel 1993). Unlike dosed DNA, GFP expression from extracellular mRNA is non-mosaic, showing that most embryonic cells have received and translated the transcript. This relatively uniform expression and low degree of mosaicism means that mRNA is well suited to studying effect on a large number of cells and for studying events in early development which start as early as 3 hpf. To demonstrate successful delivery of mRNA, a $0.50 \mu \mathrm{g} / \mu \mathrm{l}$ solution of GFP-mRNA was pipetted in the chamber and a single square pulse of $20 \mathrm{~V}$ and $50 \mathrm{~ms}$ pulse width was used to dose the pattern in Fig. 4.

Lastly, we wish to explore microfluidic modifications which allowed the devices to dose more than one compound at a time. These microfluidic devices were constructed as long hollow gold channels on a glass substrate with a $10 \times 10 \mu \mathrm{m}$ micropore etched in the roof 
(A)

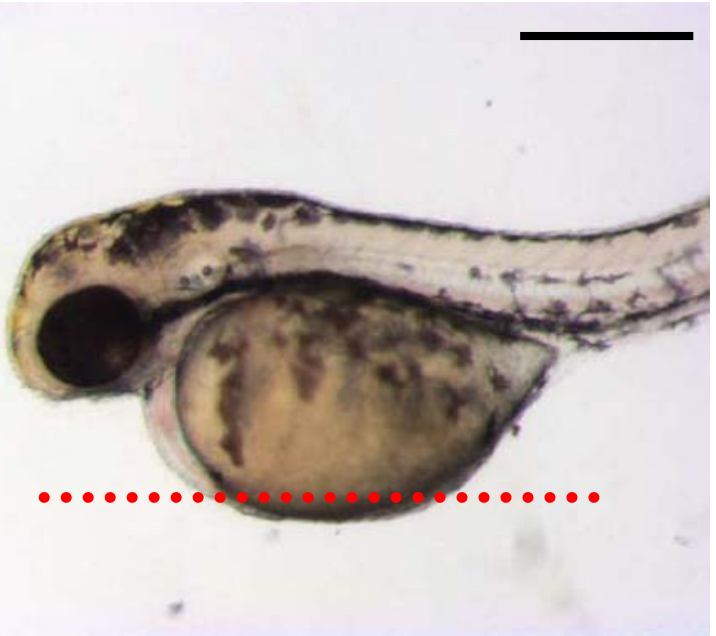

(C)

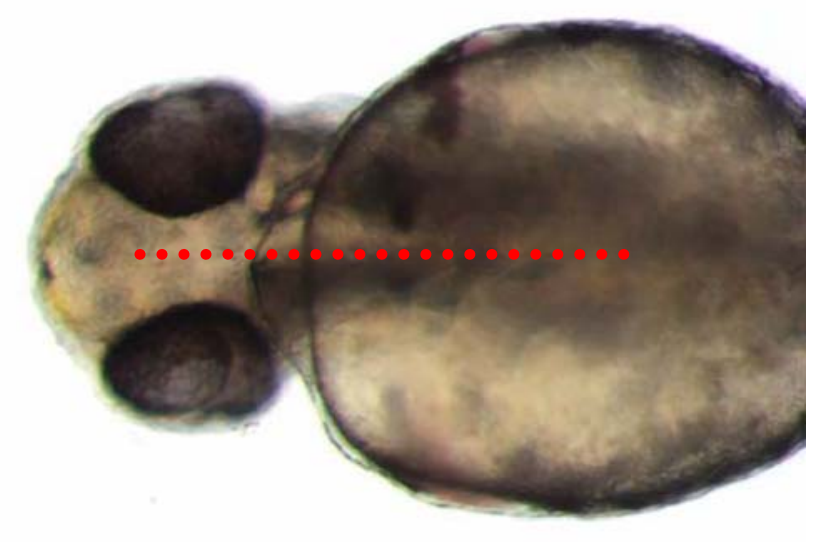

(E)

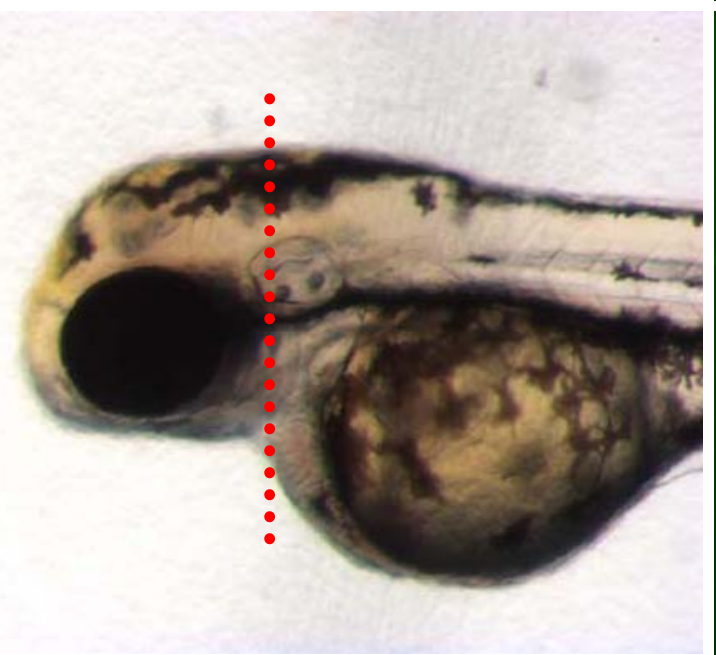

Fig. 3 Delivery of pCS2eGFP in late stage zebrafish embryos in a line pattern. (a) A $24 \mathrm{hpf}$ zebrafish was positioned on top of the cathode. A cross-section view in white light of the pCS2eGFP DNA dosed embryo is shown. The red dotted line indicates where the electrode was placed. Electrode was placed parallel to the anteroposterior axis. (b) A fluorescent image of the dosed embryo. Typical 'mosaic'
(B)

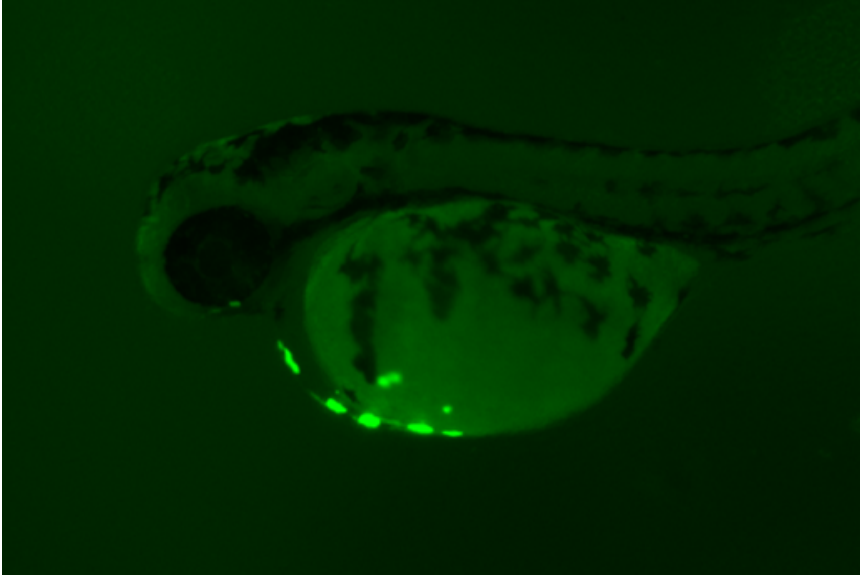

\section{(D)}
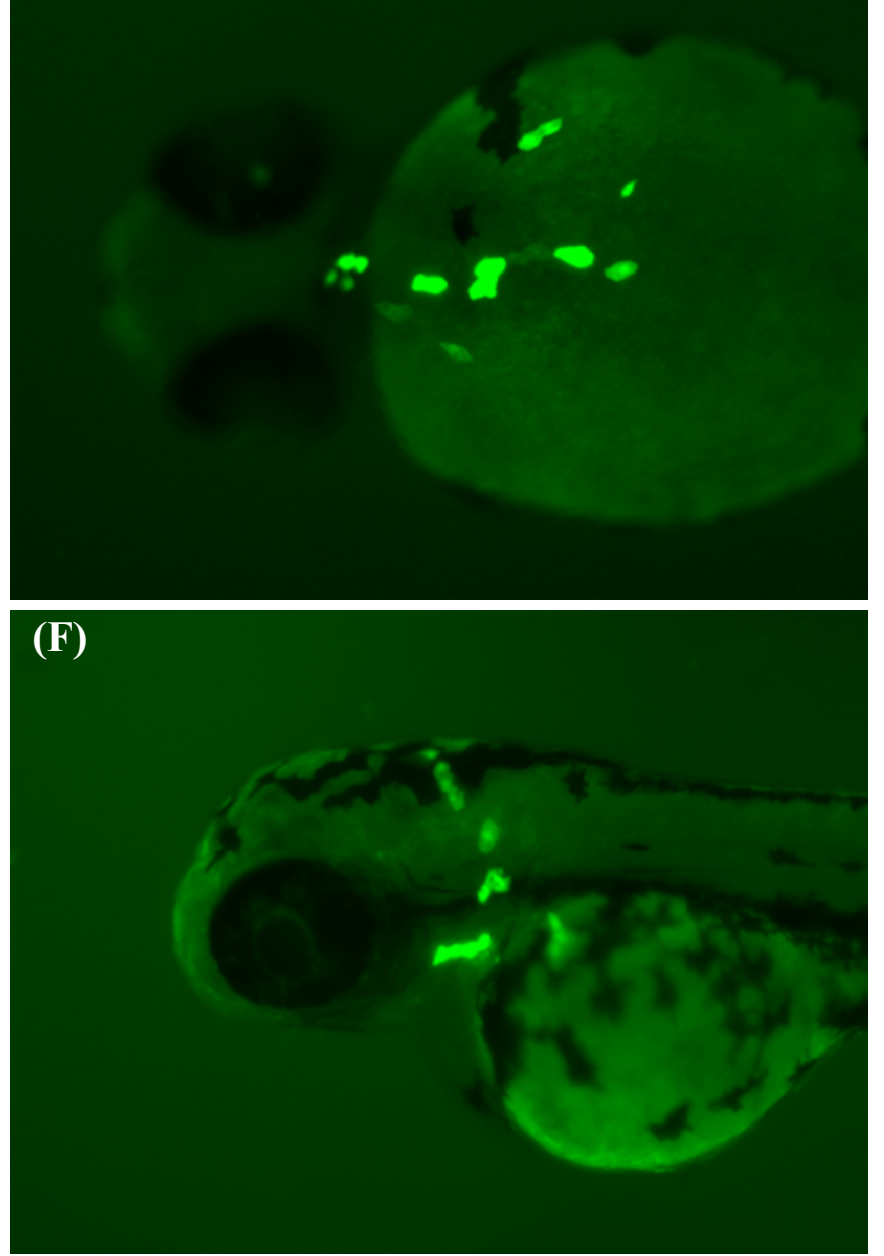

expression pattern was obtained. (c) A top-view of the same fish in normal light. (d) Fluorescent image taken from the top view. (e) White light image of a second zebrafish where the electrode was placed perpendicular to the anteroposterior axis. (f) Fluorescent image of zebrafish. Scale bar is $200 \mu \mathrm{m}$ 


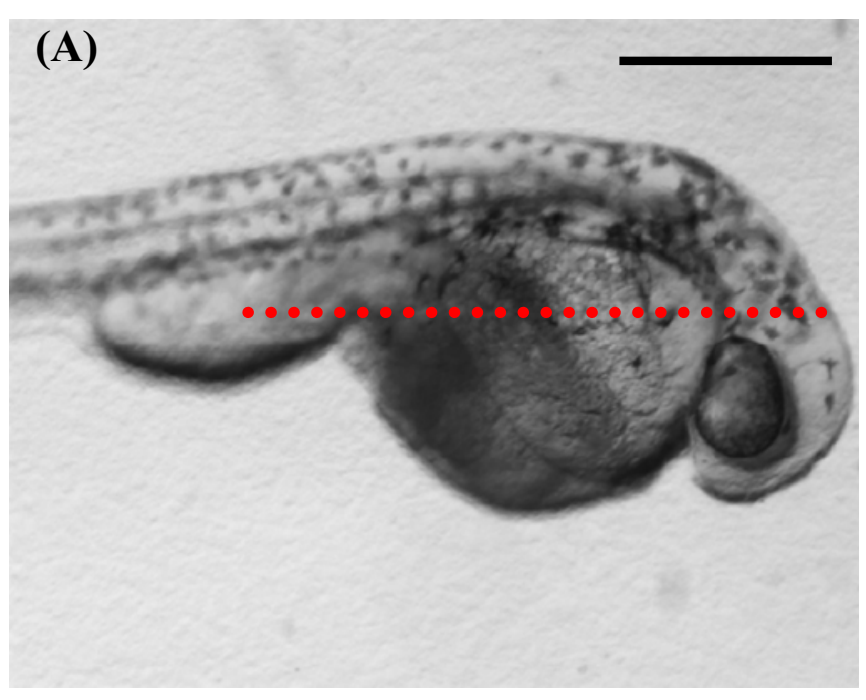

Fig. 4 GFP-mRNA was electroporated in a line shape in a $24 \mathrm{hpf}$ zebrafish. Red dotted line shows the location of electrode. Single pulse of $20 \mathrm{~V}, 50 \mathrm{~ms}$ pulse width was sufficient to deliver the mRNA;

of the channel (Supplementary Fig. 2). In this setup, the top gold channel, acted as an anode with a distant metal wire acting as cathode. Different solutions could be introduced into each channel via capillary action. In Fig. 5, a solution
(B)

$0.50 \mu \mathrm{g} / \mu \mathrm{l}$ solution was used. (a) White light image of the zebrafish (b) Fluorescent image of the zebrafish. Scale bar is $200 \mu \mathrm{m}$

of $30 \mathrm{mM}$ Texas Red and Fluoroscein Dextran mixed with E3 was introduced at the inlets and filled the channels as seen in Fig. 5(b) and (d). Embryos were positioned on top of the micropore array. A single pulse of $10 \mathrm{~V}, 100 \mathrm{~ms}$
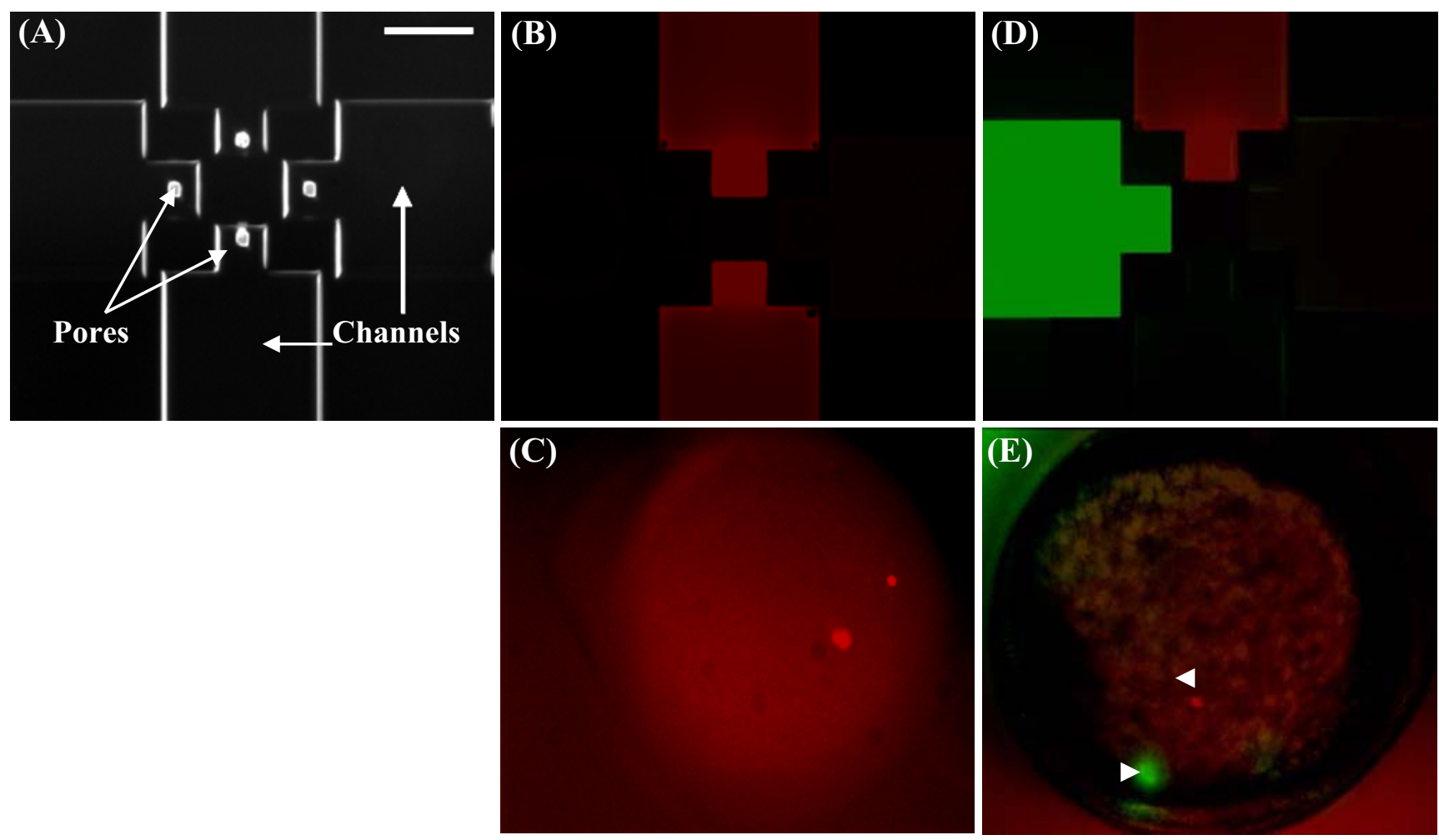

Fig. 5 A second microfluidic device of $10 \mu \mathrm{m} \times 10 \mu \mathrm{m}$ micropore arrays was fabricated. (a) Channels were fabricated as shown in Fig. S1. (b) Texas red was introduced at the inlet and filled two channels via capillary action. (c) Fluorescent image of embryo after electroporation was conducted. Two separate dosing points were clearly visible. (d) Two separate dyes: fluorescein dextran and Texas red were introduced at the inlet. (e) Two separate dosed points were visible as shown by arrowheads (ImageJ was used to superimpose two separate fluorescent dyes onto the same image). Scale bar is $200 \mu \mathrm{m}$ 
Table 1 Survival \% of zebrafish electroporated with various molecules is presented

\begin{tabular}{lclcr}
\hline Molecule & Stage (hpf) & Optimized voltage protocol & Survival \% & Delivery efficiency \\
\hline Trypan blue & $3^{\mathrm{a}}$ & $20 \mathrm{~V}, 100 \mathrm{~ms}, 2$ pulses & 80 & 60 \\
DNA & $24^{\mathrm{b}}$ & $10 \mathrm{~V}, 100 \mathrm{~ms}, 1$ pulse & 91.3 & 38 \\
mRNA & $24^{\mathrm{b}}$ & $20 \mathrm{~V}, 50 \mathrm{~ms}, 1$ pulse & 89 & 50 \\
\hline
\end{tabular}

The high survival \% can be attributed to low voltages and long pulse widths used in the experiment

${ }^{a}$ Chorionated embryos

${ }^{\mathrm{b}}$ Dechorionated embryos

pulse width was applied. Figure 5(c) and (e) show an embryo dosed via the micropore electrodes. Having a smaller molecular weight, fluorescein diffuses faster than Texas Red as seen in Fig. 5(e). The resolution of pointdosing using this method is comparable to conventional microinjection techniques. These types of pores can themselves be made to open and close mechanically, controlling diffusion of the dye into the medium (Supplementary Figs. 8 and 9).

\subsection{Survival analysis}

By visually imaging each electroporated embryo, survival and delivery efficiency of foreign molecules was determined. For chorionated embryos, only the embryos that survived $3 \mathrm{~h}$ after electroporation were deemed viable and counted. For dechorionated embryos, an embryo was only considered as having survived if the embryo was viable and it exhibited a straight anterior-posterior axis symmetry. Curved embryos were not counted and were considered non-viable. Delivery efficiency was calculated only from the survived embryos. Table 1 shows the survival and delivery efficiency of Trypan blue in chorionated embryos and GFP-DNA and GFP-mRNA into dechorionated $24 \mathrm{hpf}$ embryos under the described conditions. We believe that shorter voltages and high pulse widths than those used conventionally helped in increasing survivability and dosing efficiency respectively. Table 1 also includes other parameters such as embryo stages at which electroporation was conducted, voltage, pulse width, number of pulses and the number of embryos tested. Direct physical contact of the embryos with metal lines had minimal adverse effects on the survival of the zebrafish. Electrolytic bubbles were observed in some cases and resulted in movement of the fish due to the fish reacting to the pulse.

\section{Conclusion}

Recent zebrafish studies have shown the existence of spatial heterogeneous developmental programs which are difficult to modulate with existing experimental tools
(Lucchetta et al. 2005; Shestopalov and Chen 2008). We have demonstrated a system employing microfabricated platinum electrodes and silicone polymer capable of dosing two-dimensional spatial patterns of compounds into zebrafish. We presented the design, fabrication and results of these interfaces and demonstrated how this system could be used to 'draw' patterns of tracer molecules, mRNA and DNA into the blastula and yolk of zebrafish embryos (Danio rerio) at different stages of development. We also demonstrated an extension to the basic technology that delivers more than one compound at different locations simultaneously. We believe this technology can be a powerful tool in dynamic developmental biology studies and could provide a route towards more sophisticated multi-compound chemical interfaces for studying developmental organisms.

Acknowledgements The authors were supported by a National Science Foundation Integrated Hybrid Complex Systems (IHCS) grant and a grant from the Keck Foundation. They would also like to thank Lurie Nanofabrication Facility (LNF) at the University of Michigan for fabrication assistance; Hiroyasu Kamei and Jianfeng Zhou for their help with some of the imaging protocols, and Joay Singhal for his assistance with experimental protocols.

Open Access This article is distributed under the terms of the Creative Commons Attribution Noncommercial License which permits any noncommercial use, distribution, and reproduction in any medium, provided the original author(s) and source are credited.

\section{References}

T.C.G. Bosch, R. Augustin, K. Gellner, K. Khalturin, J.U. Lohmann, Differentiation 70, 140-147 (2002)

G.A. Cerda, J.E. Thomas, M.L. Allende, R.O. Karlstrom, V. Palma, Methods 39(3), 207-211 (2006)

B.L.M. Hogan, R.S.P. Beddington, F. Constantini, E. Lacey, Manipulating the Mouse Embryo: A Laboratory Manual (CSH Press, New York, 1994)

Y. Huang, B. Rubinsky, Biomed. Microdevices 2, 145-150 (1999)

K.-S. Huang, Y.-C. Lin, K.-C. Su, H.-Y. Chen, Biomed. Microdevices 9, 761-768 (2007) 
R.F. Ismagilov, M.M. Maharbiz, Curr. Opin. Chem. Biol. 11, 604-611 (2007)

D.A. Kane, C.B. Kimmel, Development 119, 447-456 (1993)

Y. Kawakami, A. Raya, R.M. Raya, C. Rodriguez-Esteban, J.C.I. Belmonte, Nature 435, 165-171 (2005)

M. Khine, A. Lau, C. Ionescu-Zanetti, J. Seo, L.P. Lee, Lab Chip 5, 38-43 (2005)

J.A. Kim, K. Cho, M.S. Shin, W.G. Lee, N. Jung, C. Chung, J.K. Chang, Biosens. Bioelectron. 22, 3273-3277 (2007)

V. Kohli, V. Robles, M.L. Cancela, J.P. Acker, A.J. Waskiewicz, A.Y. Elezzabi, Biotech. Bioeng. 98, 1230-1241 (2007)

E.M. Lucchetta, J.H. Lee, L.A. Fu, N.H. Patel, R.F. Ismagilov, Nature 434, 1134-1138 (2005)

P.F. Lurquin, Mol. Biotechnol. 7, 5-35 (1997)

A. Mara, J. Schroeder, C. Chalouni, S.A. Holley, Nat. Cell Biol. 9, 523-530 (2007)

K.M. Rambabu, S.H. Rao, N.M. Rao, BMC Biotechnol. 5, 362-368 (2005)
R.A.W. Rupp, L. Snider, H. Weintraub, Genes Dev. 8, 1311-1323 (1994)

A.J.H. Sale, W.A. Hamilton, Biochim. Biophys. Acta. 118, 781-788 (1967)

A.J.H. Sale, W.A. Hamilton, Biochim. Biophys. Acta. 163, 37-43 (1968)

I.A. Shestopalov, J.K. Chen, Chem. Soc. Rev. 37, 1294-1307 (2008)

M. Swartz, J. Eberhart, G.S. Mastick, C.E. Krull, Dev. Bio. 233, 13$21(2001)$

D.L. Turner, H. Weintraub, Genes Dev. 8, 1434-1447 (1994)

O. Voiculescu, F. Bertocchini, L. Wolpert, R.E. Keller, C.D. Stern, Nature 449, 1049-1052 (2007)

O. Voiculescu, C. Papanayotou, C.D. Stern, Nat. Protoc. 3, 419-426 (2008)

F.J. Vonk, M.K. Richardson, Nature 454, 282-283 (2008)

M. Westerfield, Guide for the Laboratory Use of Zebrafish (Danio rerio), 4th edn. (University of Oregon Press, Eugene, 2000)

S. Zappe, M. Fish, M.P. Scott, O. Solgaard, Lab Chip 6, 1012-1019 (2006) 